\title{
Increased expression of the pluripotency markers sex-determining region Y-box 2 and Nanog homeobox in ovarian endometriosis
}

\author{
Yong Song ${ }^{\dagger}$, Li Xiao ${ }^{\dagger}$, Jing Fu, Wei Huang ${ }^{*}$, Qiushi Wang, Xianghui Zhang and Shiyuan Yang
}

\begin{abstract}
Background: The precise etiology of endometriosis is not fully understood; the involvement of stem cells theory is a new hypothesis. Related studies mainly focus on stemness-related genes, and pluripotency markers may play a role in the etiology of endometriosis. We aimed to analyze the transcription pluripotency factors sex-determining region Y-box 2 (SOX2), Nanog homeobox (NANOG), and octamer-binding protein 4 (OCT4) in the endometrium of reproductive-age women with and without ovarian endometriosis.
\end{abstract}

Methods: We recruited 26 women with laparoscopy-diagnosed ovarian endometriosis (endometriosis group) and 16 disease-free women (control group) to the study. Endometrial and endometriotic samples were collected. SOX2, NANOG, and OCT4 expression were analyzed with quantitative real-time polymerase chain reaction, western blotting, and immunohistochemistry.

Results: Compared to the control group, SOX2 mRNA and protein expression was significantly higher in the eutopic endometrium of participants in the endometriosis group. In the endometriosis group, SOX2 and NANOG mRNA and protein expression were significantly increased in ectopic endometrium compared with eutopic endometrium; there was a trend towards lower OCT4 mRNA expression and higher OCT4 protein expression in ectopic endometrium.

Conclusions: The transcription pluripotency factors SOX2 and NANOG were overexpression in ovarian endometriosis, their role in pathogenesis of endometriosis should be further studied.

Keywords: Endometriosis, Sex-determining region Y-box 2, NANOG, Octamer-binding protein 4

\section{Background}

Endometriosis is a chronic disease characterized by the presence of ectopic endometrial implants [1]. Current treatments such as medication or surgery are effective to an extent; however, some patients experience infertility, pain from ineffective treatment, recurrence, and even malignancy. Thus, identification of the mechanisms underlying the pathogenesis of endometriosis will facilitate the development of more effective treatment for this disorder.

Despite considerable investigation, the precise etiology and pathogenesis of endometriosis is unknown. To date, the leading theories are retrograde menstruation, coelomic

\footnotetext{
*Correspondence: weihuang64@163.com

${ }^{\dagger}$ Equal contributors

Department of Obstetrics and Gynecology, West China Second University Hospital of Sichuan University, Chengdu 610041, Sichuan, P. R. China
}

metaplasia, embryonic cell rest, and lymphovascular metastasis [2]. However, none of them can annotate the pathogenesis to a point for all types of endometriosis. Current research involving stem cells should shed some light on the puzzling mechanisms of this disease [3-6]. However, data on stem or progenitor cell function in endometriosis are scarce due to the technical limitations to stem cell research. Current studies mainly focus on stemness-related genes; the pluripotency markers sexdetermining region Y-box 2 (SOX2), Nanog homeobox (NANOG), and octamer-binding protein 4 (OCT4) are the most often studied [7-9]. SOX2 is a member of the SOX (SRY-related high mobility group [HMG] box) gene family that encodes transcription factors with a single HMG DNA-binding domain [10]. SOX genes bind to the minor groove in DNA to control diverse developmental 
processes and play critical roles in cell fate determination, differentiation, and proliferation [11]. OCT4 is a member of the POU domain transcription factor family and plays a key role in the regulation of self-renewal and pluripotency in embryonic stem cells and primordial germ cells [12], while NANOG is a homeobox gene and has essential roles in maintaining self-renewal and the undifferentiated state of pluripotent stem cells [13]. In addition to maintaining the self-renewal ability of stem cells, SOX2, NANOG, and OCT4 are involved in cancer cell migration and invasion [13-15]. Aberrant SOX2, NANOG, and OCT4 expression has been demonstrated in endometriotic tissues, and OCT4 promoted endometrial cell migration activity [7-9]. However, these markers are not identified exclusively in women with ovarian endometriosis. Therefore, we aimed to examine and compare SOX2, NANOG, and OCT4 expression in endometrial or endometriotic tissues from women with and without ovarian endometriosis.

\section{Methods}

\section{Study population}

The Medical Research Review Board of West China Second University Hospital, Sichuan University (Sichuan, China) approved the study, and written informed consent was obtained from the human participants of the study. The participants were reproductive age women and had regular menstrual cycles. None had received hormonal treatment within the previous three months. Participants with adenomyosis, leiomyomas, endometrial hyperplasia, genital tumors, and acute pelvic inflammatory disease were excluded. From May 2012 to December 2012, we recruited 42 women who had undergone simultaneous laparoscopy and hysteroscopy to the study. Of these, 26 women with deep ovarian endometriosis (diameter of cyst is from $3 \mathrm{~cm}$ to $5 \mathrm{~cm}$ ) were laparoscopically diagnosed and further confirmed by pathology (endometriosis group). The r-AFS score was used for disease stage (17 stage III, and 9 stage IV). The remaining 16 (age 24-32 years, mean 27 years) were controls who had undergone simultaneous laparoscopy and hysteroscopy for infertility which had no visible evidence of endometriosis or adhesions during surgery (Table 1).

Table 1 Characteristics of study population

\begin{tabular}{lcc}
\hline Characteristics & $\begin{array}{c}\text { Endometriosis } \\
\text { group }(\mathbf{n}=\mathbf{2 6})\end{array}$ & $\begin{array}{c}\text { Non-endometriosis } \\
\text { group }(\mathbf{n}=\mathbf{1 6})\end{array}$ \\
\hline Age (yrs) & $27.94 \pm 3.45(22-35)$ & $26.90 \pm 2.47(24-32)$ \\
Body mass index $\left(\mathrm{kg} / \mathrm{m}^{2}\right)$ & $20.81 \pm 2.94$ & $19.14 \pm 2.05$ \\
Infertility duration (yrs) & $3.06 \pm 1.71$ & $3.10 \pm 1.85$ \\
r-AFS staging (n) & & \\
$\quad$ Stage III & 17 & 0 \\
$\quad$ Stage IV & 9 & 0 \\
\hline
\end{tabular}

Eutopic and ectopic endometrial tissues from ovarian endometrioma and from normal control endometrium were obtained during surgery. Of the 16 control specimens, 10 were used for quantitative real-time polymerase chain reaction (PCR) and the remaining six for western blot and immunohistochemistry analysis. Of the 26 endometriosis specimens, 13 were used for quantitative real-time PCR and the remaining 13 for western blot and immunohistochemistry analysis. Tissues were stored in a microfuge tube at $-80^{\circ} \mathrm{C}$ for PCR and western blot analysis, or immediately fixed in $10 \%$ buffered formalin and paraffin-embedded for immunohistochemical and hematoxylin and eosin staining. All participants were determined to be in the proliferative phase of their menstrual cycle as assessed by the timing of their last menstrual period and histological dating [16].

\section{RNA isolation, CDNA synthesis, and real-time PCR}

Total RNA was extracted using TRIzol according to the manufacturer's protocol (Life Technologies Inc., Carlsbad, CA, USA); the quality and concentration of purified RNA were analyzed using a NanoVue Plus spectrophotometer (Health-careBio-ScienceAB, Uppsala, Sweden). The nucleotide to protein ratios (A260:A280) of all samples were within the acceptable boundaries of 1.8 and 2.1. First-strand complementary DNA (cDNA) synthesis was performed with a PrimeScript RT Reagent Kit (TaKaRa Biotechnology, Dalian, China) used according to the manufacturer's protocol at $37^{\circ} \mathrm{C}$ for $30 \mathrm{~min}$, followed by deactivation at $85^{\circ} \mathrm{C}$ for $8 \mathrm{~s}$. PCR was performed using primers synthesized by Sangon Biotech (Shanghai, China) (Table 2). cDNA samples were diluted 10-fold for PCR. Each well of the PCR plate contained $5 \mu \mathrm{L}$ EvaGreen Supermix (Bio-Rad Laboratories, Hercules, CA, USA), $1 \mu \mathrm{L}$ of each primer $(10 \mu \mathrm{mol} / \mathrm{L})$, and $3 \mu \mathrm{L}$ diluted cDNA. Amplification was performed over 39 cycles of $95^{\circ} \mathrm{C}$ for $30 \mathrm{~s}, 95^{\circ} \mathrm{C}$ for $5 \mathrm{~s}$, and $60^{\circ} \mathrm{C}$ for $10 \mathrm{~s}$; melting curve analysis from $65^{\circ} \mathrm{C}$ to $95^{\circ} \mathrm{C}$ at a rate of $5 \mathrm{~s}$ per step was performed to determine data quality using a CFX96 RealTime PCR Detection System (Bio-Rad Laboratories). Only data with single melting peaks were included in the final analysis. All experiments were performed in triplicate. The threshold cycle values were normalized to the threshold value of human glyceraldehyde-3-phosphate dehydrogenase $(G A D P H)$ and the results expressed as the mean \pm SD.

\section{Immunohistochemistry}

Immunohistochemical staining was performed using monoclonal mouse anti-human SOX2 (ab75485, Abcam, Cambridge, MA, USA), polyclonal rabbit anti-human NANOG (ab80892, Abcam), and rabbit anti-human 
Table 2 Primer sequences used in quantitative real-time PCR

\begin{tabular}{llll}
\hline Gene & Sense primer $\mathbf{5}^{\prime} \mathbf{- 3}^{\prime}$ & Antisense primer $\mathbf{5}^{\prime} \mathbf{- \mathbf { 3 } ^ { \prime }}$ & Genbank accession $\mathbf{N M}$ \\
\hline GAPDH & TGCACCACCAACTGCTTAGC & GGCATGGACTGTGGTGATGAG & NM_002046 \\
SOX2 & TACAGCATGTCCTACTCGCAG & GAGGAAGAGGTAACCACAGGG & NM_003106 \\
NANOG & AAGGTCCCGGTCAAGAAACAG & CTTCTGCGTCACACCATTGC & NM_024865 \\
OCT4 & GCAGCGACTATGCACAACGA & CCAGAGTGGTGACGGAGACA & NM_002701 \\
\hline
\end{tabular}

OCT4 (ab18976, Abcam) as primary antibodies. Briefly, serial sections were prepared, mounted on gelatin-coated slides, dried overnight at $37^{\circ} \mathrm{C}$, deparaffinized in xylene, and rehydrated through a graded ethanol series. To retrieve the epitopes, the slides were immersed in citrate antigen retrieval buffer ( $\mathrm{pH}$ 6) for $10 \mathrm{~min}$ at $120^{\circ} \mathrm{C}$. After cooling, the sections were incubated with $3 \% \mathrm{H}_{2} \mathrm{O}_{2}$ for 10 min to block endogenous peroxidase activity, blocked with $10 \%$ normal goat serum for $15-30 \mathrm{~min}$, then incubated with the primary antibodies (1:100 SOX2, 1:400 NANOG, 1:100 OCT4) at $4^{\circ} \mathrm{C}$ overnight. Secondary biotinylated antibody and streptavidin-peroxidase conjugate were applied according to the manufacturer's instructions (Beijing Zhongshan Biotech, Beijing, China); staining was visualized with diaminobenzidine, and then the sections were counterstained with hematoxylin and mounted. Colorectal carcinoma tissues were used as positive controls. Negative controls were performed by incubating sections with phosphate-buffered saline instead of primary antibodies.

\section{Western blot analysis}

Total proteins were collected using radioimmunoprecipitation lysis buffer (P0013B, Beyotime Biotechnology, Shanghai, China) according to the manufacturer's instructions. Protein concentration was determined using a bicinchoninic acid assay kit (Beyotime Biotechnology). Protein $(30 \mathrm{ng}$ ) from each specimen underwent 10\% sodium dodecyl sulfate-polyacrylamide gel electrophoresis and was transferred to polyvinylidene fluoride membranes (Millipore, Billerica, MA, USA). The membranes were blocked for $1 \mathrm{~h}$ in $5 \%$ defatted milk at room temperature. Subsequently, the membranes were incubated with monoclonal mouse anti-human SOX2 (1:200; ab75485, Abcam), polyclonal rabbit anti-human NANOG (1:300; ab80892, Abcam), OCT4 (1:500; ab18976, Abcam), and rabbit

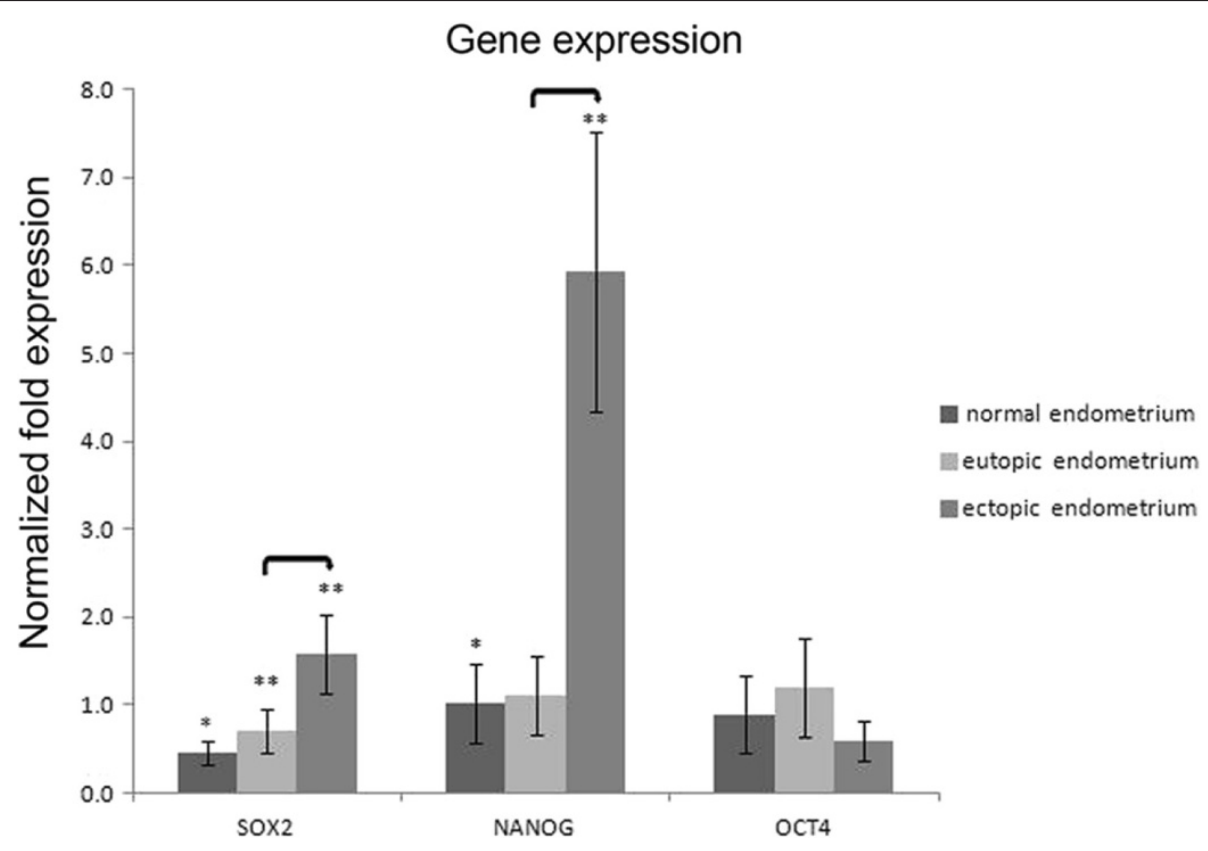

Figure 1 Quantitative real-time PCR analysis of SOX2, NANOG, and OCT4 mRNA. Analysis was carried out on normal endometrium ( $\mathrm{n}=10$ ) and paired eutopic and ectopic endometrium specimens $(n=13)$. Double-asterisk values are significantly different from single-asterisk values. Asterisks denote significant differences between eutopic or ectopic endometrium and normal endometrium. Connecting lines denote significant differences between eutopic and ectopic endometrium. $P<0.05$ was significant. Error bars denote SEM. 
polyclonal anti-GAPDH (1:10000; bs-2188R, Bioss, Beijing, China) antibodies overnight at $4{ }^{\circ} \mathrm{C}$. Then, the membranes were incubated with horseradish peroxidase-conjugated secondary antibody for $1 \mathrm{~h}$ at room temperature. Proteins were detected with a chemiluminescence kit (Millipore) and the strength of the bands was analyzed with ImageJ $2 x$ (National Institutes of Health, Bethesda, MD, USA). Protein levels were normalized to that of the internal control GAPDH.

\section{Statistical analysis}

Statistical analysis was performed using SPSS version 18.0 (SPSS, Chicago, IL, USA). All data are expressed as the mean \pm SD. Differences between groups were evaluated with one-way analysis of variance with a post hoc test (Student-Newman-Keuls method); $P<0.05$ was considered statistically significant (two-tailed).

\section{Results}

SOX2, NANOG, and OCT4 mRNA expression

SOX2 mRNA expression in the eutopic endometrium of ovarian endometriosis were significantly higher than that in normal endometrium $(P=0.02)$; expression of NANOG and OCT4 mRNA in eutopic endometrium increased but no statistically significant when compared with normal controls. When compared to eutopic endometrium, SOX2 and NANOG mRNA expression in ectopic endometrium was significantly increased $(P=0.004 ; P=0.01$, respectively),

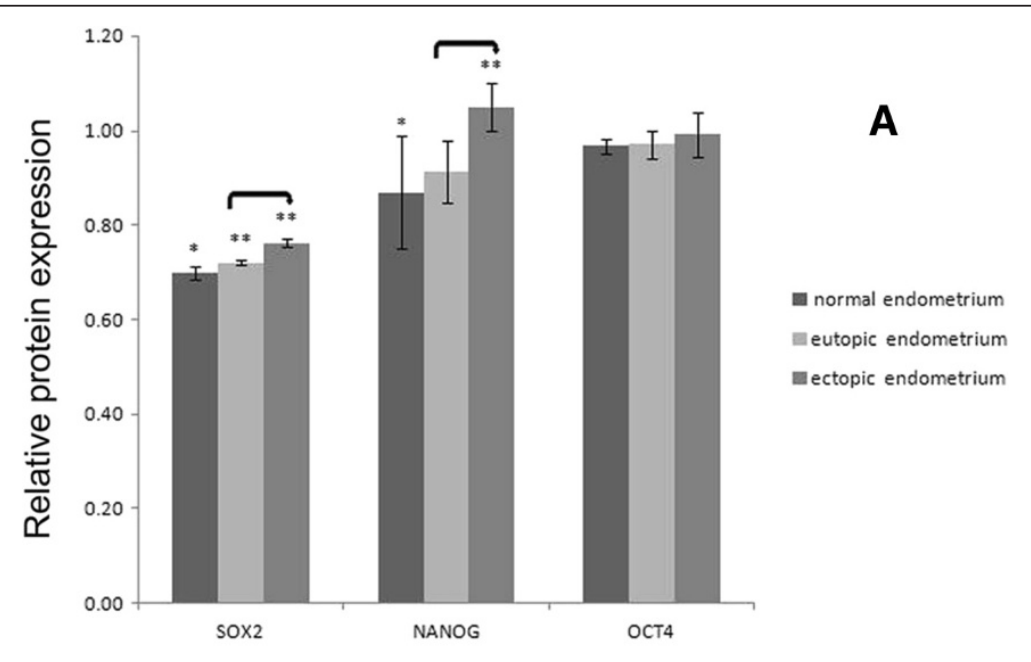

B

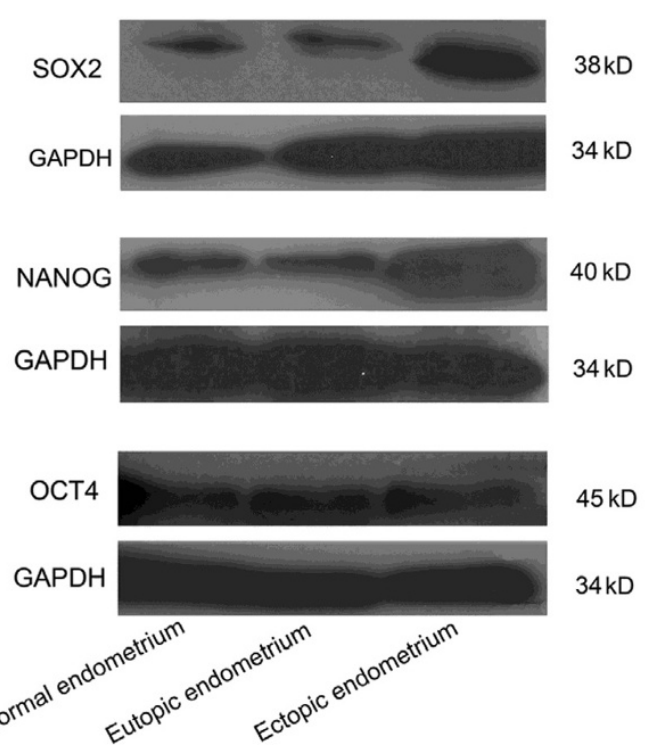

Figure 2 Western blot analysis of SOX2, NANOG, and OCT protein. (A) Relative levels of SOX2, NANOG, and OCT4 protein in normal endometrium $(n=6)$ and paired eutopic and ectopic endometrium $(n=13)$. Double-asterisk values are significantly different from single-asterisk values. Asterisks denote significant differences between eutopic or ectopic endometrium and normal endometrium. Connecting lines denote significant differences between eutopic and ectopic endometrium. $P<0.05$ was significant. Error bars denote SEM. (B) Representative western blot of SOX2, NANOG, and OCT4 protein. GAPDH, Internal control. 
but OCT4 mRNA expression in ectopic endometrium tended to be lower $(P=0.05)$. Both SOX2 and NANOG mRNA expression in ectopic endometrium were significantly higher than that in normal endometrium $(P=0.001$; $P=0.009$, respectively), and only OCT4 mRNA expression in ectopic endometrium tended to be lower $(P>0.05)$ (Figure 1).

\section{Expression and location of SOX2, NANOG, and OCT4 protein}

Compared to normal endometrium, SOX2 protein expression in the eutopic and ectopic endometrium of ovarian endometriosis was significantly increased $(P=0.04 ; P=0.009$, respectively); NANOG protein expression in ectopic endometrium was statistically significantly different $(P=0.04)$; OCT4 protein expression in eutopic and ectopic endometrium tended to be higher $(P>0.05)$. Both SOX2 and NANOG protein expression in ectopic endometrium were significantly higher than that in eutopic endometrium $(P=0.01 ; P=0.007$, respectively), while only OCT4 protein expression in ectopic endometrium tended to be higher $(P>0.05)$ (Figure 2).

SOX2, NANOG, and OCT4 protein were mainly expressed in the nuclei of glandular epithelial or stromal cells of

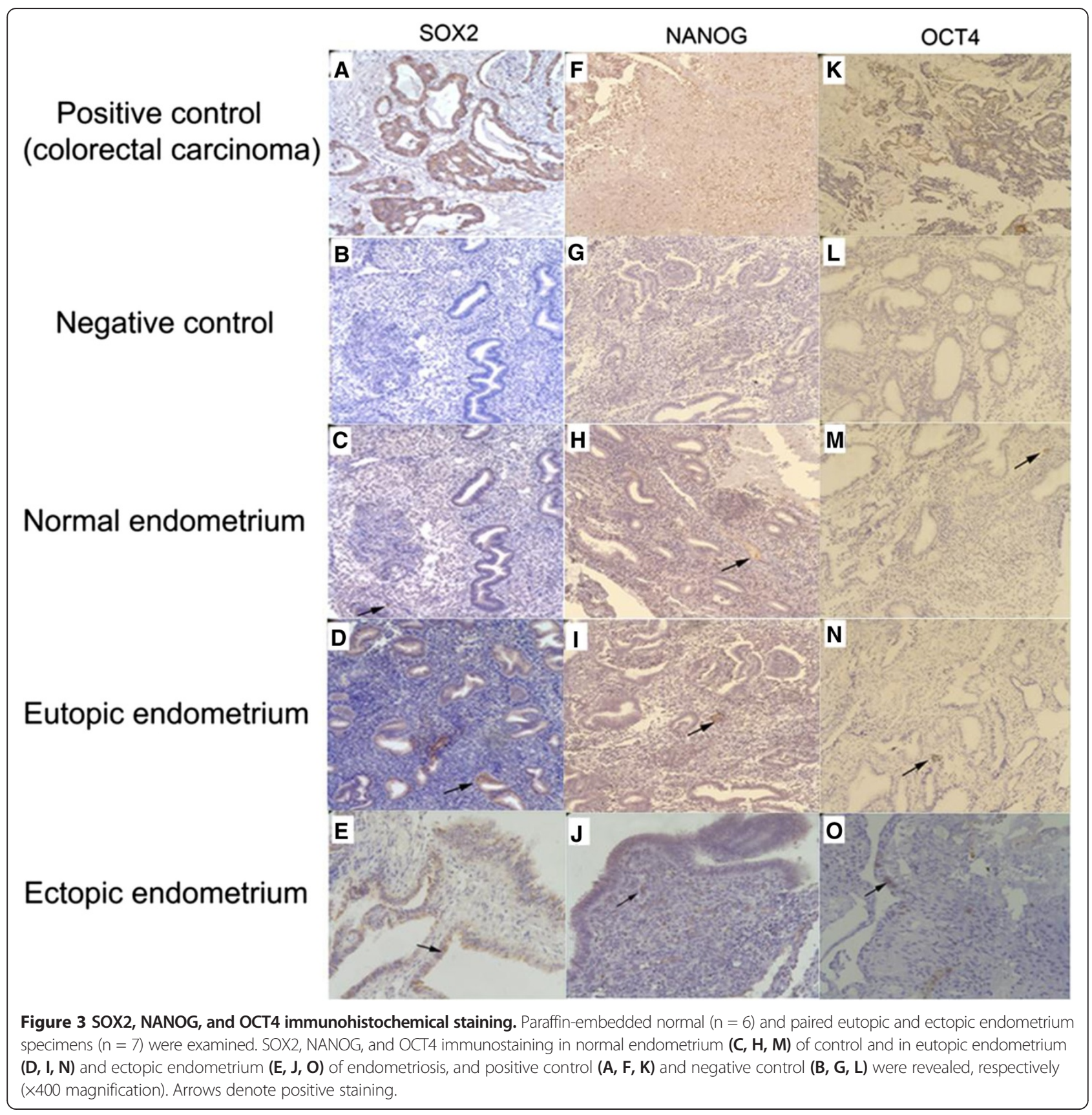


ectopic and eutopic endometrium of ovarian endometriosis and normal control endometrium, with low expression detected in the cytoplasm of both cell types (Figure 3).

\section{Discussion}

SOX2 and OCT4 are transcription factors essential for maintaining the self-renewal and pluripotent phenotype of embryonic stem cells [12,17]. Previous studies have revealed that they are also key factors in the reprogramming of somatic cells to a pluripotent state [18]. Similarly, NANOG can maintain the pluripotency and self-renewal characteristics of embryonic stem cells [19]. It has also been verified that these factors are involved in cancer cell migration and invasion $[13,15,20]$. Several other studies have also demonstrated that they are expressed in human normal endometrium, endometriotic tissues, and in endometrial cancer [7-9,21].

Emerging evidence suggests that there is decreased apoptosis, increased proliferation, and higher migration and invasion ability in the eutopic endometrium of endometriosis $[22,23]$. The aberrant SOX2 expression in ovarian endometriosis in our study, consistent with that reported previously $[7,8]$, may indicate a stem cell origin of endometriosis. As a pluripotent transcription factor, SOX2 overexpression in eutopic and ectopic endometrial specimens of ovarian endometriosis was expected to result in increased proliferation and inhibit apoptosis by targeting apoptosis-related proteins as it does in neural stem cells and prostate cancer $[24,25]$. As SOX2 affected migration/ invasion by downregulating matrix metalloproteinase- 2 in colorectal cancer [15], we believe that its overexpression in ovarian endometriosis may be a potential mechanism that leads to the intrinsic differentiation of eutopic endometrial cells from that in normal endometria, and play an important role in the disease pathogenesis.

In this study, NANOG expression was significantly increased in the ectopic endometrium of women with ovarian endometriosis. This result differs from that of previous studies $[7,9]$. The reason may be related to the study population, the ages of the participants, or their menstrual cycles.

Endometriosis is usually considered an estrogen-dependent disorder [1]. In ectopic endometrium, estradiol (E2) is overproduced by aromatase and is not metabolized because the ectopic endometrium lacks $17 \beta$-hydroxysteroid dehydrogenase type 2 activity. Two recent studies support the positive link between high levels of E2 and SOX2 or NANOG expression [26,27]. SOX2 and NANOG overexpression may foster self-renewal and increase cell survival in ovarian endometrial tissues that can further facilitate ectopic tissue growth. More importantly, data from large cohort studies indicate that endometriosis patients have an increased risk of ovarian cancer [28,29]. Recently, two studies revealed that SOX2 overexpression and advanced ovarian cancer were closely associated [30,31]. Another study demonstrated that NANOG was involved in ovarian tumorigenesis through migration and invasion [13]. In this study, NANOG expression in ectopic endometrium was nearly six times that in eutopic endometrium, and SOX2 expression was four times in ectopic endometrium. The results indicate that NANOG alone or in combination with SOX2 may contribute to the transformation of ovarian endometriosis to ovarian cancer.

In our study, OCT4 expression was similar among normal endometrium and eutopic and ectopic endometrium of endometriosis. It has been established that OCT4 is a key transcription factor in the regulation of self-renewal and pluripotency in embryonic stem cells and in the reprogramming of somatic cells to a pluripotent state. Chang et al. found that OCT4 was upregulated in human ectopic endometriotic tissues and that it contributed to ectopic endometrial growth by stimulating endometrial cell migration activity [9]. However, we could not verify this in the present study, which might have been due to the study population and the menstrual cycles of the participants; therefore, further studies would require a larger sample size.

In our study, there were some drawback like sample size is not large and amount of endometrial tissue cannot match all experimental items that result in some index not reach statistical significance. Infertile women are chosen as control is not perfect, however, it's hard to get endometrial tissue from normal fertile women that undergo laparoscopy and hysteroscopy procedure. A larger sample size and the functional research of SOX2 and NANOG in pathogenesis of endometriosis is anticipated in our further study.

\section{Conclusions}

In reproductive-age women with ovarian endometriosis, the transcriptional factor SOX2 and NANOG are over expression. Future studies is need to determine their role in pathogenesis of ovarian endometriosis.

\section{Abbreviations \\ E2: Estradiol; MMP: Matrix metalloproteinases; OCT4: Octamer-binding protein 4; SOX2: Sex-determining region Y-box 2. \\ Competing interests \\ The authors declare that they have no competing interests. \\ Authors' contributions \\ YS designed the study, performed experiments and drafted the manuscript. LX performed experiments, did statistic analysis and drafted the manuscript. JF and QW designed the study and performed experiments. WH designed the study and helped to draft the manuscript. XZ and SY collected the tissue samples and participated in the study. All authors read and approved the final manuscript.}

\section{Acknowledgements}

This work was supported by a grant from the Science and Technology Bureau of Sichuan (2012SZ0030). We are grateful to Dr. Dan Zhang for his help in sample collection. 
Received: 18 December 2013 Accepted: 3 May 2014

Published: 18 May 2014

\section{References}

1. Giudice LC, Kao LC: Endometriosis. Lancet 2004, 364:1789-1799.

2. Macer ML, Taylor HS: Endometriosis and infertility: a review of the pathogenesis and treatment of endometriosis-associated infertility. Obstet Gynecol Clin North Am 2012, 39:535-549.

3. Chan RW, Schwab KE, Gargett CE: Clonogenicity of human endometrial epithelial and stromal cells. Biol Reprod 2004, 70:1738-1750.

4. Schwab KE, Chan RW, Gargett CE: Putative stem cell activity of human endometrial epithelial and stromal cells during the menstrual cycle. Fertil Steril 2005, 84:1124-1130.

5. Sasson IE, Taylor HS: Stem cells and the pathogenesis of endometriosis. Ann N Y Acad Sci 2008, 1127:106-115.

6. Oliveira FR, Dela Cruz C, Del Puerto HL, Vilamil QT, Reis FM, Camargos AF: Stem cells: are they the answer to the puzzling etiology of endometriosis? Histol Histopathol 2012, 27:23-29.

7. Forte A, Schettino MT, Finicelli M, Cipollaro M, Colacurci N, Cobellis L, Galderisi U: Expression pattern of stemness-related genes in human endometrial and endometriotic tissues. Mol Med 2009, 15:392-401.

8. Gotte M, Wolf M, Staebler A, Buchweitz O, Kiesel L, Schuring AN: Aberrant expression of the pluripotency marker SOX2 in endometriosis. Fertil Steril 2011, 95:338-341.

9. Chang JH, Au HK, Lee WC, Chi CC, Ling TY, Wang LM, Kao SH, Huang YH, Tzeng CR: Expression of the pluripotent transcription factor OCT4 promotes cell migration in endometriosis. Fertil Steril 2013, 99:1332-1339.

10. Avilion AA, Nicolis SK, Pevny LH, Perez L, Vivian N, Lovell-Badge R: Multipotent cell lineages in early mouse development depend on SOX2 function. Genes Dev 2003, 17:126-140.

11. Fang X, Yu W, Li L, Shao J, Zhao N, Chen Q, Ye Z, Lin SC, Zheng S, Lin B: ChIP-seq and functional analysis of the SOX2 gene in colorectal cancers. OMICS 2010, 14:369-384.

12. Kehler J, Tolkunova E, Koschorz B, Pesce M, Gentile L, Boiani M, Lomeli H, Nagy A, McLaughlin KJ, Scholer HR, Tomilin A: Oct4 is required for primordial germ cell survival. EMBO Rep 2004, 5:1078-1083.

13. Siu MK, Wong ES, Kong DS, Chan HY, Jiang L, Wong OG, Lam EW, Chan KK, Ngan HY, Le XF, Cheung AN: Stem cell transcription factor NANOG controls cell migration and invasion via dysregulation of E-cadherin and FoxJ1 and contributes to adverse clinical outcome in ovarian cancers. Oncogene 2013, 32:3500-3509.

14. Chiou SH, Wang ML, Chou YT, Chen CJ, Hong CF, Hsieh WJ, Chang HT, Chen YS, Lin TW, Hsu HS, Wu CW: Coexpression of Oct4 and Nanog enhances malignancy in lung adenocarcinoma by inducing cancer stem cell-like properties and epithelial-mesenchymal transdifferentiation. Cancer Res 2010, 70:10433-10444.

15. Han X, Fang X, Lou X, Hua D, Ding W, Foltz G, Hood L, Yuan Y, Lin B: Silencing SOX2 induced mesenchymal epithelial transition and its expression predicts liver and lymph node metastasis of CRC patients. PLoS One 2012, 7:e41335

16. Noyes RW, Hertig AT, Rock J: Dating the endometrial biopsy. Am J Obstet Gynecol 1975, 122:262-263.

17. Fong $\mathrm{H}$, Hohenstein KA, Donovan PJ: Regulation of self-renewal and pluripotency by Sox2 in human embryonic stem cells. Stem Cells 2008 26:1931-1938.

18. Takahashi K, Tanabe K, Ohnuki M, Narita M, Ichisaka T, Tomoda K, Yamanaka S Induction of pluripotent stem cells from adult human fibroblasts by defined factors. Cell 2007, 131:861-872.

19. Rodda DJ, Chew JL, Lim LH, Loh YH, Wang B, Ng HH, Robson P: Transcriptional regulation of nanog by OCT4 and SOX2. J Biol Chem 2005, 280:24731-24737.

20. Dai X, Ge J, Wang X, Qian X, Zhang C, Li X: OCT4 regulates epithelial-mesenchymal transition and its knockdown inhibits colorectal cancer cell migration and invasion. Oncol Rep 2013, 29:155-160.

21. Zhou X, Zhou YP, Huang GR, Gong BL, Yang B, Zhang DX, Hu P, Xu SR: Expression of the stem cell marker, Nanog, in human endometrial adenocarcinoma. Int J Gynecol Pathol 2011, 30:262-270.

22. Dmowski WP, Ding J, Shen J, Rana N, Fernandez BB, Braun DP: Apoptosis in endometrial glandular and stromal cells in women with and without endometriosis. Hum Reprod 2001, 16:1802-1808.
23. Taniguchi F, Kaponis A, Izawa M, Kiyama T, Deura I, Ito M, Iwabe T, Adonakis G, Terakawa N, Harada T: Apoptosis and endometriosis. Front Biosci (Elite Ed) 2011, 3:648-662.

24. Lin F, Lin P, Zhao D, Chen Y, Xiao L, Qin W, Li D, Chen H, Zhao B, Zou H, Zheng $X$, Yu X: Sox2 targets cyclinE, p27 and survivin to regulate androgen-independent human prostate cancer cell proliferation and apoptosis. Cell Prolif 2012, 45:207-216.

25. Feng R, Zhou S, Liu Y, Song D, Luan Z, Dai X, Li Y, Tang N, Wen J, Li L: Sox2 protects neural stem cells from apoptosis via up-regulating survivin expression. Biochem J 2013, 450:459-468.

26. Zhang Y, Eades G, Yao Y, Li Q, Zhou Q: Estrogen receptor alpha signaling regulates breast tumor-initiating cells by down-regulating miR-140 which targets the transcription factor SOX2. J Biol Chem 2012, 287:41514-41522.

27. Yang L, Luo L, Ji W, Gong C, Wu D, Huang H, Liu Q, Xia B, Hu G, Zhang W, Zhang Q, Liu J, Zhang W, Zhuang Z: Effect of low dose bisphenol A on the early differentiation of human embryonic stem cells into mammary epithelial cells. Toxicol Lett 2013, 218:187-193.

28. Borgfeldt $C$, Andolf $E$ : Cancer risk after hospital discharge diagnosis of benign ovarian cysts and endometriosis. Acta Obstet Gynecol Scand 2004 83:395-400.

29. Kobayashi H, Sumimoto K, Moniwa N, Imai M, Takakura K, Kuromaki T, Morioka E, Arisawa K, Terao T: Risk of developing ovarian cancer among women with ovarian endometrioma: a cohort study in Shizuoka, Japan. Int J Gynecol Cancer 2007, 17:37-43.

30. Ye F, Li Y, Hu Y, Zhou C, Chen H: Expression of Sox2 in human ovarian epithelial carcinoma. J Cancer Res Clin Oncol 2011, 137:131-137.

31. Zhang J, Chang DY, Mercado-Uribe I, Liu J: Sex-determining region Y-box2 expression predicts poor prognosis in human ovarian carcinoma. Hum Pathol 2012, 43:1405-1412.

doi:10.1186/1477-7827-12-42

Cite this article as: Song et al:: Increased expression of the pluripotency markers sex-determining region Y-box 2 and Nanog homeobox in ovarian endometriosis. Reproductive Biology and Endocrinology 2014 12:42.

\section{Submit your next manuscript to BioMed Central and take full advantage of:}

- Convenient online submission

- Thorough peer review

- No space constraints or color figure charges

- Immediate publication on acceptance

- Inclusion in PubMed, CAS, Scopus and Google Scholar

- Research which is freely available for redistribution
C Biomed Central 